\title{
The Use of an Intraoperative Skeletal Traction Device for Delayed Reduction in Pelvic Fractures in Two Dogs
}

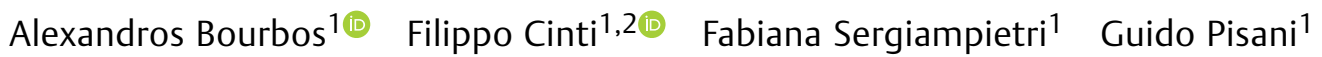 \\ ${ }^{1}$ Department of Surgical, Centro Veterinario Pisani, Carli, Chiodo, \\ Luni, Italy \\ ${ }^{2}$ Department of Surgical, Eastcott Referrals, Edison Business Park, \\ Swindon, United Kingdom

\begin{abstract}
Address for correspondence Alexandros Bourbos, DVM, MRCVS, Centro Veterinario Pisani, Carli, Chiodo, Via Palmiro Togliatti 8, 19034 Luni, Italy (e-mail: alxbourbos@yahoo.gr).
\end{abstract} \\ VCOT Open 2020;3:e40-e45. \\ Abstract \\ Keywords \\ - pelvic fractures \\ - canine \\ - skeletal traction \\ device \\ Two large-breed dogs underwent delayed surgical repair of pelvic fractures with the aid of a \\ skeletal traction device. Distraction and reduction in the fractures using the skeletal traction \\ device were monitored by direct visualization and intraoperative fluoroscopy. Postopera- \\ tive radiographs revealed good to near-anatomical bone reduction and fracture alignment \\ without narrowing of the pelvic canal. Both dogs had complete functional recovery with no \\ evidence of neurological deficits. The use of a skeletal traction device should be considered \\ for reduction and fixation of delayed pelvic fractures.
}

\section{Introduction}

Pelvic fractures are common in small animals constituting $16 \%$ of all fractures in dogs and $25 \%$ of all fractures in cats. ${ }^{1}$ Although the cause of pelvic fractures is often not known, road traffic accidents are considered the most common reason in both species. Other high-energy traumatic incidents such as crush injury or falling from a height account for some pelvic injuries. ${ }^{2}$ Young animals are overrepresented and this may be associated with roaming behavior. ${ }^{2,3}$ Anatomically the pelvis may be thought of as a 'box-like structure' with struts running through its interior and supported by a large muscle mass. ${ }^{4}$ Because of this rigid structure, displacement of fragments is not usually seen unless there are at least two or often three fractures, which commonly occur in specific locations and follow several predictable patterns. ${ }^{5}$ Fractures involving the pubic symphysis are the exception and may result in avulsion/fracture of the ischial tuberosity or iliac wing, or non-displaced stress fractures. ${ }^{4}$ The nature of pelvic fractures necessitates thorough evaluation of organs and organ systems. In a study of dogs involved in road traffic accidents, $71 \%$ had injuries to multiple organs, including pulmonary, soft tissue, spinal and urinary tract trauma, as well as haemoabdomen and cardiac dysrhythmias. ${ }^{6}$ In particular, cranial or craniomedial displacement of the iliac bone has been associated with damage to the L6 and L7 nerve roots and injury to the lumbosacral trunk. Thus, the timing for repair of pelvic fractures depends largely on the degree of collateral injuries, and surgery often has to be delayed until the animal is stabilized and able to undergo general anaesthesia. ${ }^{7}$ In animals with lifethreatening injuries, surgical repair may be delayed for up to 7 to 10 days after injury. ${ }^{4}$ After this time, muscle contraction and early fibrosis at the fracture site render adequate fracture reduction difficult or impossible. ${ }^{4}$ The aim of this case report was to introduce and evaluate the use of a skeletal traction device for the delayed repair of pelvic fractures that would not have been difficult to reduce using conventional techniques.

\section{Case Report}

Two dogs were referred for pelvic fractures; case 1 suffered a road traffic accident, whereas in case 2 , non-unions were present as complications of previous bilateral double pelvic osteotomy. Case 1 was a 9-year-old, mixed-breed dog weighing $30 \mathrm{~kg}$ and case 2 was a 6 -month-old, Bernese Mountain dog weighing $35 \mathrm{~kg}$. At the time of referral, the fractures had been present for 12 days in case 1 and 14 days in case 2 . The results of physical and neurological examinations, haematological and serum biochemical analyses, of thoracic and abdominal radiography and ultrasonography were within normal limits.
License terms Stuttgart · New York

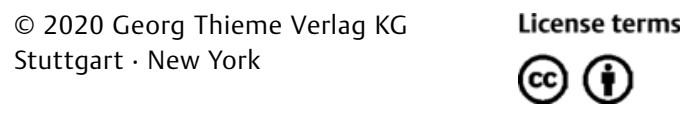

DOI https://doi.org/

10.1055/s-0040-1713124. ISSN 2625-2325.
February 18, 2020

accepted

April 29, 2020 
Ventrodorsal and lateral radiographic views of the pelvis were obtained in both cases. Case 1 had bilateral fractures, which included a long oblique fracture of the left wing of the ilium, fracture of the pubis and transverse fracture of the right iliac body. Case 2 had undergone bilateral double pelvic osteotomy and had in addition a fracture of the right ischial table; the complications were associated with implant failure due to the use of undersized screws and also the fact that the screw orientation was perpendicular to the plate and not divergent to resist the pull out.

\section{Surgical Technique}

The anaesthetic protocols were based on individual patient requirements and included methadone (Semfortan, Dechra, Italy) ( $0.3 \mathrm{mg} / \mathrm{kg}$, intramuscularly]) and meloxicam (Metacam, Boehringer Ingelheim, Italy) $(0.2 / \mathrm{mg} / \mathrm{kg}$, intramuscularly) for perioperative analgesia. Cefazoline (Teva, Italy) $(22 \mathrm{mg} / \mathrm{kg}$, intravenously) was administrated 30 minutes preoperatively and every 90 minutes thereafter for a total of three doses during the surgical procedure. General anaesthesia was induced with propofol (Propovet, Zoetis, Italy) $(2-4 \mathrm{mg} / \mathrm{kg}$, intravenously) and maintained with $2 \%$ isoflurane (Piramal Critical Care, West Drayton, UK) in oxygen. Intraoperative analgesia was provided with methadone. Both dogs were placed in lateral recumbency with the forelimbs secured to the operating table. A hanging limb technique was used, and the surgical site was aseptically prepared for surgery.

In case 1 , the most displaced fragment was treated first to facilitate reduction on the opposite site. Fluoroscopy was used intraoperatively to guide and confirm reduction in the fractures. The skeletal traction device, commercially available (AdMaoira, Reggio Emilia, Italy: https://www.admaiora.eu/en/), is L-shaped, and the long component has a micrometric movement that allowed stand elongation. One end of the stand was secured to the operating table rail with clamps. The short component consisted of a stainless-steel tube for attachment of the tie-band ( - Fig. 1). A small stab incision was made over the ischial tuberosity on both sides, and a $3 \mathrm{~mm}$ Steinman pin (Alcyon Srl, Cherasco, Italy) was carefully introduced on each side perpendicular to the long axis of the pelvis while avoiding the nerves and vascular supply of the region. The pins were inserted to a distance of $\sim 2 \mathrm{~cm}$ from the caudal edge of the ischial tuberosity to avoid possible fracture at this site, which was considered the anchorage point (-Fig. 2). A sterilized tie-band was looped at the base of each of the pin-skin interfaces to evaluate the feasibility of traction and to apply equal traction on both sides of the pelvis ( $\mathbf{- F i g . 3 C}$ ). The forelimbs were secured to the edge of the operating table in a cranial direction to counteract the traction forces and to avoid translation; injury to the patient was avoided as described for long bone traction, paying attention that during the traction the pins do not bend and create soft tissue damage. ${ }^{8,9}$ This attachment was considered the opposition point. After a second aseptic preparation of the surgical field, a standard lateral approach to the iliac body was done. The load required for distraction of the fractured segment was applied by an operating room assistant who increased the load at the surgeon's request with 5-minute rest intervals between each load increase until visual and palpable reduction was evident and confirmed by fluoroscopy ( - Fig. 3D). Distraction force to the two bilateral Steinmann pins was applied simultaneously to avoid translation of the opposite side and deformation of pelvic conformation. Load application for reduction was performed by turning the handle on the traction device in a clockwise direction, increasing the length of the long component of the L-shaped stand. During the procedure, contamination of the surgical field was prevented because the caudal end of the device and the assistant were outside the surgical field and

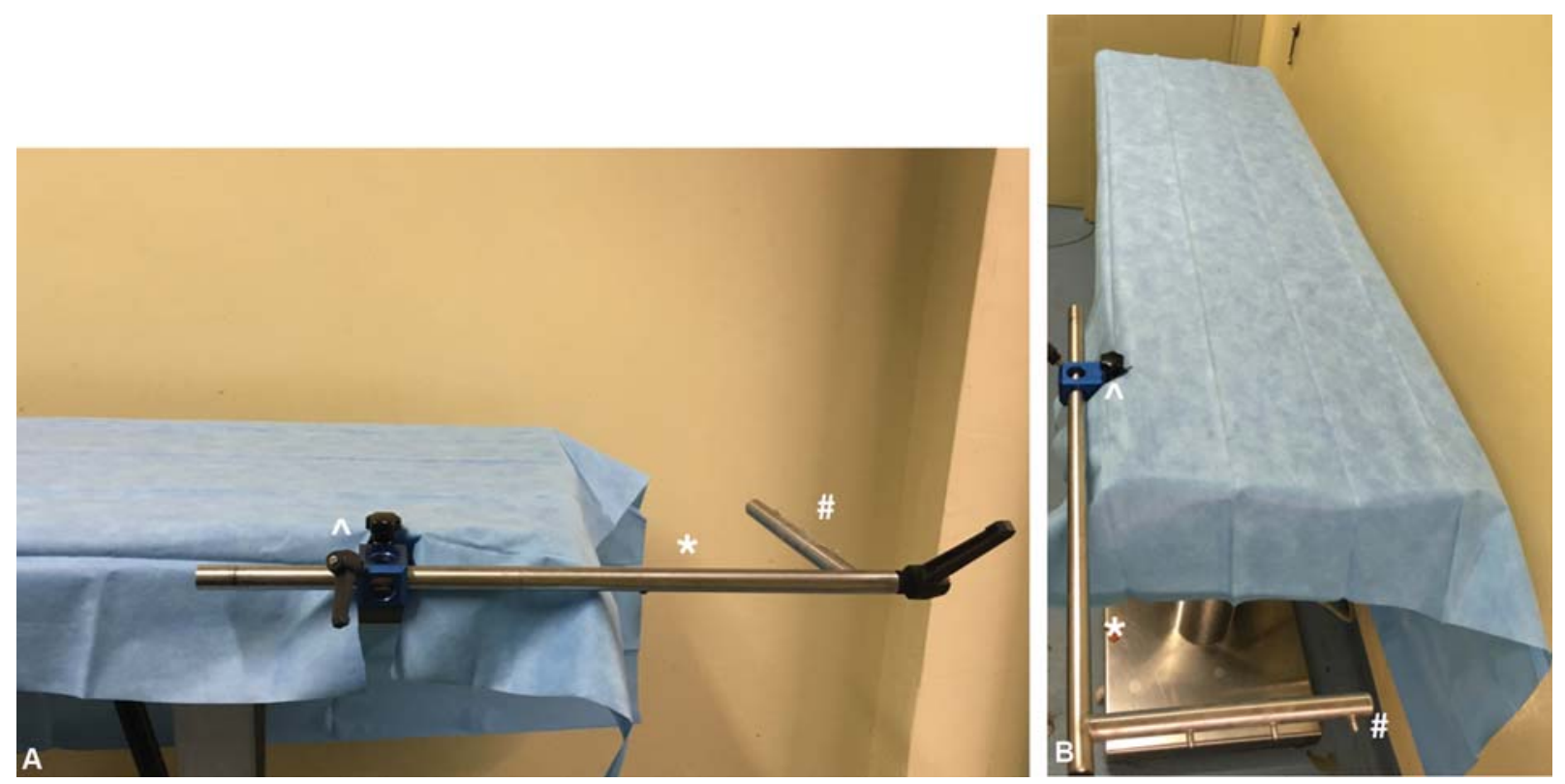

Fig. 1 The L-shaped skeletal traction device mounted on the operating table $(\mathrm{A}, \mathrm{B})$. The device has a long $\left({ }^{*}\right)$ and a short component (\#) and a clamp $\left({ }^{\wedge}\right)$ for attaching the device to the table. 

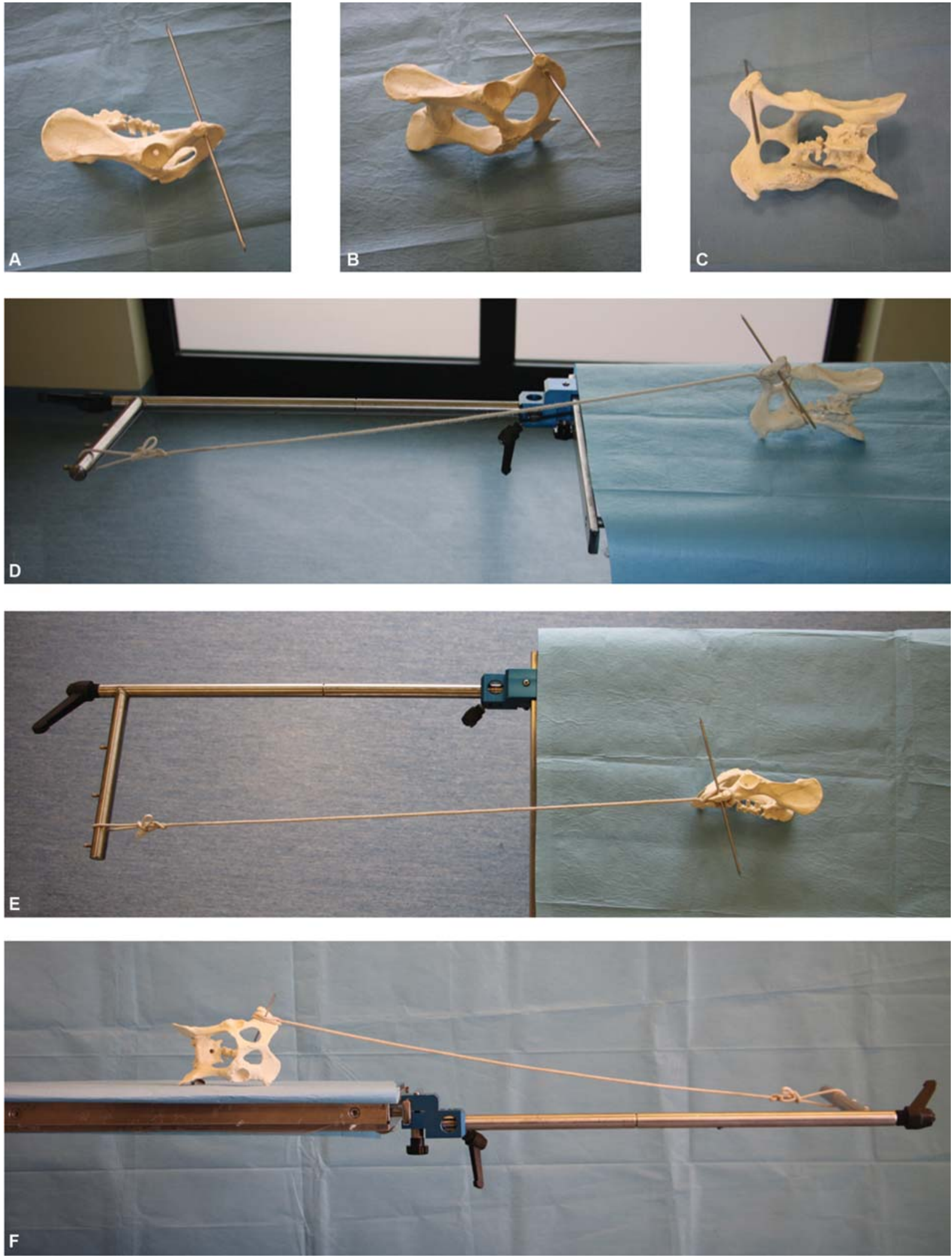

Fig. 2 Bone model showing the position of the Steinmann pin, on the ischiatic tuberosity, in lateral, ventral and dorsal views (A-C). The connection between the pin and the skeletal traction device is also shown (D-F).

the portion of the device closest to the surgical field was covered by the surgical drapes. Once the fracture on the right side had been reduced, a 3.5 -mm locking bone plate (Fixin, InTrauma, Rivoli, Italy) was applied; after wound closure, the procedure was repeated on the left side after re-positioning the dog in right lateral recumbency. Postoperative radiographs were obtained to score alignment and apposition as anatomical, near-anatomical, good, fair or poor $^{10}$ and 

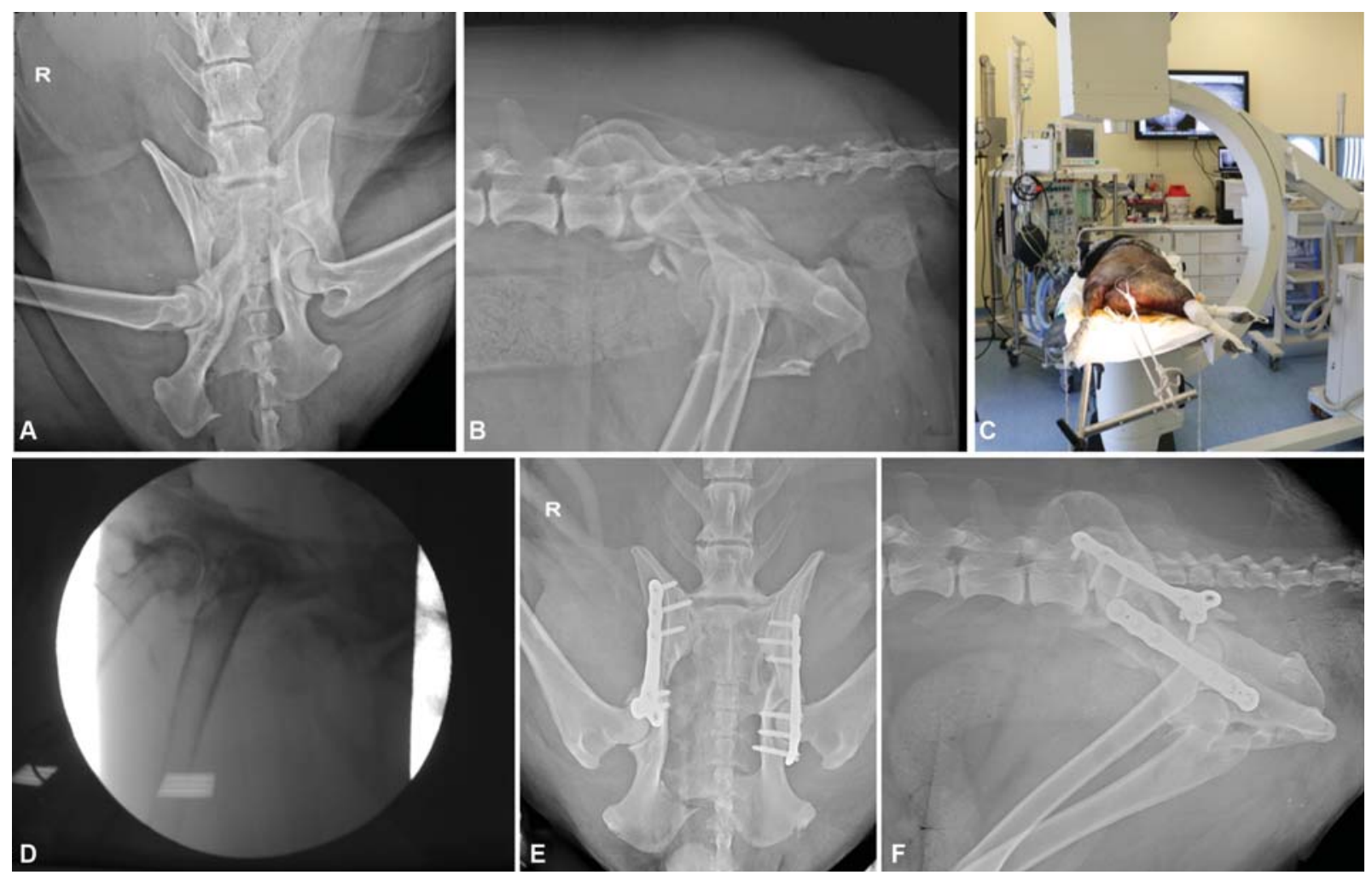

Fig. 3 Ventrodorsal and lateral radiographic views of the pelvis in case 1 showing a long oblique fracture of the left ilial wing, fracture of the pubis and transverse fracture of the right iliac body with fracture of the right ischiatic table (A, B). Preoperative bilateral Steinmann pin placement in the ischial tuberosities and their connection to the skeletal traction device (C). Perioperative fluoroscopic image used to estimate the degree of distraction of the pelvis (D). Postoperative radiographic view showing proper implant positioning with no narrowing of the pelvic canal (E, F).

confirmed proper implant positioning (-Fig. 3E, F), also the alignment of the bone segment was considered good to nearanatomical without evidence of pelvic canal narrowing.

In case 2 , a staged surgery, 2 days apart, was performed to minimize surgical time and the risk of infection. For the first intervention, the patient was placed in left lateral recumbency to reduce and repair the iliac body fracture on the right. All surgical steps involving placement of the 3-mm Steinman pins (Alcyon Srl) were done using the technique described in case 1 . After removal of the double pelvic osteotomy plate and screws, traction was applied until reduction was confirmed visually and by means of palpation and fluoroscopic examination. The fracture was stabilized with two 3.5-mm locking plates (Fixin) placed parallel to one another. Postoperative radiographs showed proper implant placement and near-anatomical bone alignment ( - Fig. 4E, F). The iliac body fracture on the left side was repaired 2 days later using the same surgical technique, with postoperative radiographs revealing good alignment and no narrowing of the pelvic canal. Both dogs had methadone and meloxicam for 48 hours postoperatively and received tramadol ( Formevet, Italy), gabapentin (Pfizer, Italy) and meloxicam orally for 14 days after the discharge.

\section{Discussion}

Fractures of the pelvis occur most commonly in the ilium, ${ }^{11}$ and sacroiliac fracture/luxation is a significant source of pain and instability and the most common pelvic injury associated with neurological deficits. ${ }^{12}$ In human as well as in veterinary surgery, pelvic fractures require adequate reduction and stabilization to control pain and to reduce blood loss. ${ }^{7,13,14}$ Pelvic fractures can pose two potential problems requiring surgical intervention: significant narrowing of the pelvic canal by the caudal fragment, sometimes with compromise of the urinary bladder and large intestines; and trauma to the lumbosacral nerve trunk, which branches into the sciatic nerve just medial to the midbody of the ilium. ${ }^{15}$ Early aggressive fracture management using a combination of internal and external fixation techniques has become the gold standard in human medicine. Although attempts to provide fracture fixation within 24 hours of injury is a first-line approach, particularly for femur fractures, this strategy has been questioned for pelvic fractures, which are often accompanied by multiple injuries. ${ }^{16,17}$ It currently appears that, in human medicine, the safest window for internal fixation is 6 days to 2 weeks after injury when the systemic inflammatory response has subsided and before callus formation limits reduction. ${ }^{18}$ Traction tables are commonly used in human trauma patients and standardized reproducible techniques are routinely used for fracture reduction. These techniques include proper patient positioning, specific instrumentation and application of intraoperative skeletal traction. The rationale behind the use of a skeletal traction device is to counteract muscle contraction to facilitate reduction in the bone fragments and regain normal limb length. The entire application and reduction in the described 

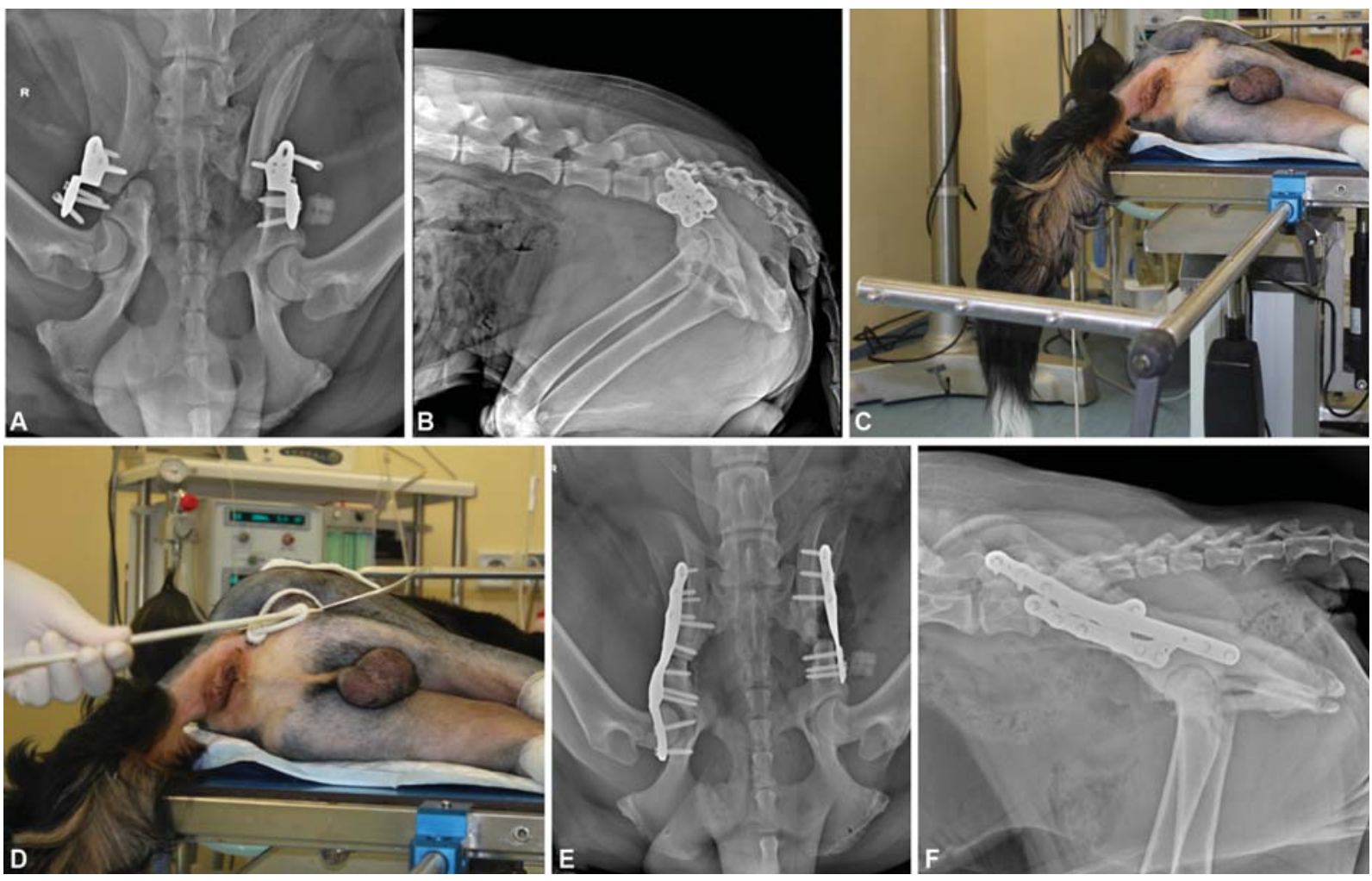

Fig. 4 Ventrodorsal and lateral radiographic views of the pelvis in case 2 showing implant failure on the right side, with plate pull-out and failure of the plate screws on the left side (A, B). Preoperative placement of a Steinmann pin and its connection to the skeletal traction device (C, D). Postoperative radiographic view of successful reduction and fixation of the left and right iliac body fracture using locking plates (E, F).

fractures using a skeletal traction device are based on the ligamentotaxis technique with the goal of using tension for alignment of bone fragments. This technique could possibly be used to facilitate specific minimally invasive surgical procedures. We found that the use of a skeletal traction device intraoperatively greatly facilitated reduction in the bone fragments before application of a plate. To our knowledge, there are no studies on the use of a skeletal traction device for the treatment of pelvic fractures in veterinary medicine. Rovesti et al reported excellent results after using a skeletal traction device for long bone fracture reduction and repositioning of misaligned bone fragments associated with the fracture pattern or bone segment traction in dogs. ${ }^{8}$ In the present study, reduction and realignment of the bone fragments were achieved through coordination by both, the primary surgeon working intraoperatively to manipulating the bone fragments and the assistant moving the short segment of the traction device. Complications such as impairment of vascular and nerve integrity attributable to excessive traction were not encountered. In human medicine, neurological damage associated with application of skeletal traction has been reported in patients with no neurological signs before traction. ${ }^{19}$ However, the interval between the initial injury and surgical intervention appeared to impact on the duration and force of traction needed to realign the bone fragments. Although the application of anchorage and opposition point appears to be straightforward, slippage of those points could potentially result in tissue trauma. Moreover, if the procedure requires prolonged traction, it may be advisable to temporarily use bone clamps to maintain reduction thereby allowing reduction in the load exerted by the skeletal traction device and preservation of tissue perfusion. Once reduction and stabilization are achieved with bone plates, the traction load may be modified by the surgeon. We suppose that young patients with delayed fracture repair may require more force during skeletal traction because of rapid callus formation at the fracture site. Preparation and installation of the traction system may appear timeconsuming, but the reduction in surgical time during bone apposition and osteosynthesis was comparable to the time needed for a conventional surgical procedure time of $\sim 60$ to 90 minutes. In human medicine, a dual pelvic sling used to treat pelvic fractures provided effective suspension, traction and compression when applied to the iliac crest and trochanteric regions. The force of traction can be varied by adjusting the degree of lateral displacement of each sling, the direction of pull and the pulley-weight arrangement. ${ }^{20}$ However, this type of skeletal traction depends on the nature of the fracturedislocation and the degree and direction of displacement determined on anteroposterior, inlet and tangential radiographic views. ${ }^{21}$ In our cases, the only parameter of substantial importance was the presence of an intact ischial tuberosity connected to the iliac body, which provided the anchorage point for pin insertion and traction. The indications for using a skeletal traction device in our cases differed from those cited in a previous study ${ }^{8}$; we used the device to overcome muscle contraction, which resulted from the delayed nature of the fracture repair, to facilitate open reduction and internal fixation. Our findings emphasize that longer standing pelvic 
fractures in dogs constitute complex injuries that are often difficult to manage surgically. The limitations of this study are on one hand inherent to a two-case report and on the other, to the relative lack of experience of the authors with regard to the forces required for skeletal traction. Evaluations are ongoing by the authors to better estimate the traction force, times and intervals necessary to reduce and align segments in various pelvic fracture patterns, as well as overall surgery times. In conclusion, this report shows that using a skeletal traction device greatly facilitated reduction and stabilization of longer standing pelvic fractures in two dogs.

\section{Conflict of Interest}

None declared.

\section{References}

1 Phillips IR. A survey of bone fractures in the dog and cat. J Small Anim Pract 1979;20(11):661-674

2 Hill FW. A survey of bone fractures in the cat. J Small Anim Pract 1977;18(07):457-463

3 Denny HR. Pelvic fractures in the dog: a review of 123 cases. J Small Anim Pract 1978;19(03):151-166

4 Moens N, DeCamp C. Fractures of the pelvis. In: Johnston S, Tobias KM, eds. Veterinary Surgery Small Animal. Second edition. Missouri, St Louis: Elsevier; 2018:938-956

5 Messmer M, Montavon PM. Pelvic fractures in the dog and cat: a classification system and review of 556 cases. Vet Comp Orthop Traumatol 2004;17:167-183

6 Streeter EM, Rozanski EA, Laforcade-Buress Ad, Freeman LM, Rush JE. Evaluation of vehicular trauma in dogs: 239 cases (JanuaryDecember 2001). J Am Vet Med Assoc 2009;235(04):405-408

7 Peterson NW, Buote NJ, Barr JW. The impact of surgical timing and intervention on outcome in traumatized dogs and cats. J Vet Emerg Crit Care (San Antonio) 2015;25(01):63-75

8 Rovesti GL, Margini A, Cappellari F, Peirone B. Clinical application of intraoperative skeletal traction in the dog. Vet Comp Orthop Traumatol 2006;19(01):14-19
9 Peirone B, Rovesti GL, Baroncelli AB, Piras L. Minimally invasive plate osteosynthesis fracture reduction techniques in small animals. Vet Clin North Am Small Anim Pract 2012;42(05): 873-895, v

10 Breashears LA, Fitch RB, Wallace LJ, Wells CS, Swiderski K. The radiographic evaluation of repaired canine ilial fractures. Vet Comp Orthop Traumatol 2004;17:64-72

11 DeCamp CE. Fractures of the pelvis. In: Johnson AL, Houlton JEF, Vannini R, eds. AO Principles of Fracture Management in Dog and Cat. Davos Platz, Switzerland: AO Publishing; 2005:161-199

12 DeCamp C. Brinker, Piermattei, and Flo's Handbook of Small Animal Orthopedics and Fracture Repair. Fifth edition. Missouri, St Louis: Elsevier; 2016:433

13 Peltier LF. Complications associated with fractures of the pelvis. J Bone Joint Surg Am 1965;47(05):1060-1069

14 Papanastasopoulos K, Myriokefalitakis E, Drougas Th, Savidis K, Kateros K, Sarantos K. Major complications of pelvic fractures. Orthop Proc Online Febr 2018;41 Available at: https://online. boneandjoint.org.uk/doi/abs/10.1302/0301-620X.93BSUPP_III.0 930345e

15 Harasen G. Pelvic fractures. Can Vet J 2007;48(04):427-428

16 Browner BD, Cole JD, Graham JM, Bondurant FJ, Nunchuck-Burns SK, Colter HB. Delayed posterior internal fixation of unstable pelvic fractures. J Trauma 1986;26(04):325-333

17 Pape HC, van Griensven M, Rice J, et al. Major secondary surgery in blunt trauma patients and perioperative cytokine liberation: determination of the clinical relevance of biochemical markers. J Trauma 2001;50(06):989-1000

18 Fulkerson EW, Egol KA. Timing issues in fracture management: a review of current concepts. Bull NYU Hosp Jt Dis 2009;67(01): 58-67

19 Albracht BG, Jenkins MD. Sciatic nerve palsy after distal femoral traction for vertically unstable pelvic fracture in a neurologically intact patient. J Am Acad Orthop Surg Glob Res Rev 2019;3 (09): $\mathrm{e} 045$

20 Holm CL. Treatment of pelvic fractures and dislocations. Skeletal traction and the dual pelvic traction sling. Clin Orthop Relat Res 1973;(97):97-107

21 Dunn AW, Morris HD. Fractures and dislocations of the pelvis. J Bone Joint Surg Am 1968;50(08):1639-1648 